Nutr. Metabol. 1971;13:71-72

\title{
Buchbesprechungen - Book Reviews - Livres nouveaux
}

Nutrition et economic familiale. FAO of the United Nations, Rome 1968. Terminology Bulletin No 16. PU: TB/16. 87 p.

II s'agit d'un dictionnaire donnant l'équivalence des 620 mots anglais, français, espagnols et allemands de la nutrition et de l'alimentation.

II apparaît clairement combien les mots clés sont intraduisibles, car recouvrant des réalités différentes. Le «food» anglais n'est pas Гéquivalent de $\Gamma$ «aliment» français, encore beaucoup plus charge de symboles et de tonus émotif.

Food aid: aide alimentaire. Food projection: projections alimentaires. Les «tra-ductions» ne seront valables que si les genres de vie et conception de la vie deve-naient communs. Prenons acte du sens de cette tentative.

J. Trémolières, Paris

Nutrition in Preschool and School Age. VII. Symposium der Schwedischen Stiftung für Ernährung. Almqvist \& Wiksells, Uppsala 1969.154 pp., zahlreiche Abb. und Graphiken.

Von namhaften Forschern in Tylösand, Schweden, im August 1968 in englischer Sprache gehaltene Vorträge samt Diskussionsvoten liegen in gediegener Ausstattung vor. Der erste von J. M. Tanner, dem bekannten Wachstumsforscher, hat das Wachstum des Kindes in Industriezentren zum Thema. - Ernährung und Zellwachs-tum werden von M. Winick behandelt, dann die chemische Zusammensetzung beim Heranwachsenden von B. Fr $\pi$ s-Hansen.

Zur engeren Thematik gehören die folgenden Vorträge: B. Lindquist über Nahrungsbedarf im Vorschul- und Schulalter, G. Sterky über spezielle Ernäh-rungsprobleme während der Pubertät und St. Sjölin über Ernährungserhebuagen bei Kindern und Jugendlichen.

Von Vahlquist wird die körperliche Entwicklung nach frühkindlicher Mangel-ernährung behandelt, währenddem J. Cravioto et al. die 1965 erstmals beschrie-benen deletären psychischen Folgen erörtern. - Fragen des Energiehaushalts während des Wachstums bespricht J. Mayer, die heute so wesentlichen psychologischen und sozialen Aspekte der Essgewohnheiten sind Thema des Vortrages von J. Yudkin.

L. Anderfelt bespricht die Bedeutung der Nahrungsmittelindustrie, um Er-nährungssitten zu beeinflussen, St. Skerfving diskutiert die zunehmend wichtige Frage der Mahlzeiten in der Schule.

Kostvorschriften in Tagesheimen bilden Gegenstand des Vortrages von B. Gor-nicki, dasselbe Thema wird für Schulspeisungen von N. Eeg-Larsen behandelt.

Ernährungsbedürfnisse des behinderten Kindes werden schliesslich von B. Isaks-son dargestellt, und das Symposium endet mit dem Referat von N. Hallman und R. Kantero über kindliche Ernährung und Gesundheit des Erwachsenen.

Das hier vermittelte in humanen Erhebungen und tierexperimentellen Unter-suchungen gewonnene Wissen umfasst im zur Verfügung stehenden Rahmen das ganze Spektrum moderner Ernährungsphysiologie von Art und Menge der Energie-spender bis zu den in Abhängigkeit von Alter und Geschlecht zu empfehlendeo Wirkstoff- und Mineralmengen. 
Dem Interessierten vermittelt der dünne Band eine Fülle von Informationen, die ansonst nur nach Konsultation zahlreicher Fachbücher zu erhalten sind.

Ritzel, Basel

72 Buchbesprechungen - Book Reviews - Livres nouveaux

K. Lang: Biochcmie der Ernährung. 2., erw. und neubearb. Aufl. Steinkopf, Darmstadt 1970. XVI + 694 pp., 43 fig., 347 tab. Ganzl. DM 180.-.

In der 2.Auflage dieses Standardwerkes hat der Autor die wesentlichen Ergebnisse der Emährungsforschung der letzten Jahre berücksichtigt und in kritischer Auswahl eine beachtliche Zahl von neuen Befunden aufgenommen. Dies erforderte eine gründliche Überarbeitung des Textes und wesentliche Ergänzungen, teils in Form neuer Kapitel über die natürliche Radioaktivität der Nahrung und des menschlichen Organismus, die radio-aktive Kontamination von Lebensmítteln und über «nicht ganz harmlose Substanzen» in der Nahrung. Die grosszügige Ausstattung mit Abbildungen und Tabellen ist einer ge-naueren Information sehr dienlich. Durch diese Erweiterungen hat jedoch der transparente Aufbau dieses Buches keineswegs gelitten. Die umfassende Literaturübersicht, eine präzise Beschreibung wichtiger Details und die gekonnte Darstellung der engen Beziehungen zwischen Ernährung und Stoffwechsel machen das Buch für jeden, der mit wissenschaft-lichen Fragen dieser Art in Berührung kommt, besonders wertvoll. Dem kritischen Leser tritt beim Studium dieses Buches klar vor Augen, dass ein erschreckend grosser Teil un-serer Kenntnisse auf diesem Gebiet nur auf dem Experiment am Tier beruht und im Be-reich der Ernährung und des Stoffwechsels des Menschen noch grössere Lücken zu füllen sind. Dieser Aspekt einer Bestandsaufnahme ist nicht das geringste Verdienst dieses Buches. G. Wolfram, München 